\title{
Regional brain activity during tasks devoted to the central executive of working memory
}

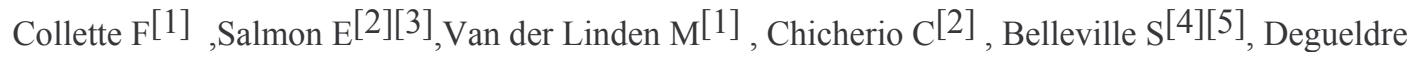 \\ $\mathrm{C}^{[2]}$, Delfiore $\mathrm{G}^{[2]}$, Franck $\mathrm{G}^{[2][3]}$ \\ [1] Neuropsychology Unit, University of Liège, Liège, Belgium \\ [2] Cyclotron Research Centre, University of Liège, Liège, Belgium \\ [3] Department of Neurology, University of Liège, Liège, Belgium \\ [4] Research Centre, University Geriatric Institute of Montreal, Montreal, Canada \\ [5] Research Group in Experimental Neuropsychology, University of Montreal, Montreal, Canada
}

\begin{abstract}
Most previous PET studies investigating the central executive (CE) component of working memory found activation in the prefrontal cortex. However, the tasks used did not always permit to distinguish precisely the functions of the $\mathrm{CE}$ from the storage function of the slave systems. The aim of the present study was to isolate brain areas that subserve manipulation of information by the CE when the influence of storage function was removed. A PET activation study was performed with four cognitive tasks, crossing conditions of temporary storage and manipulation of information. The manipulation of information induced an activation in the right (BA 10/46) and left (BA 9/6) middle frontal gyrus and in the left parietal area (BA7). The interaction between the storage and manipulation conditions did not reveal any significant changes in activation. These results are in agreement with the hypothesis that $\mathrm{CE}$ functions are distributed between anterior and posterior brain areas, but could also reflect a simultaneous involvement of controlled (frontal) and automatic (parietal) attentional systems. In the other hand, the absence of interaction between the storage and manipulation conditions demonstrates that the CE is not necessarily related to the presence of a memory load.
\end{abstract}

Keywords: Working memory; Central executive; PET

\section{Introduction}

According to Baddeley [2], working memory refers to a limited capacity system which is responsible for the temporary storage and processing of information. This model comprises a modality-free controlling central executive and a number of peripheral slave systems ensuring temporary maintenance of information. Among these slave systems, the phonological loop has been the most thoroughly explored. This system provides temporary storage for speech-based material and is composed of two subsystems: a passive phonological input store and an active articulator/ rehearsal process. The central executive (CE) is assumed to be an attentional control system responsible for strategy selection, control and co-ordination of various processing tasks.

Numerous functional neuroimaging studies have investigated the neural substrates of the different subcomponents of working memory. With regard to the phonological loop, a network of left-sided areas was found by various authors [1,4,31,36,37]. More precisely, the phonological store was localised in the left supramarginal gyrus and the articulatory rehearsal system in Broca's area. Other activated regions were the supplementary motor area, premo-tor cortex, anterior cingulate, insula, thalamus and cerebellum. Activation was also found in the para-hippocampal gyrus $[5,31,36]$ and it was plausibly related to an intervention of long-term memory in span tasks [20]. On the other hand, some studies showed unilateral or bilateral activation of the prefrontal cortex (BA 9/46) during the performance of tasks involving the central executive [8,24,32,33,37]. However, activation of other regions, such as the posterior parietal cortex, premotor areas and inferior frontal cortex was also found in these tasks. A major difficulty in the exploration of the neural substrate of the central executive is that a wide range of cognitive functions has been ascribed to that system. Recently, Baddeley [3] distinguished different functions of that system, such as the capacity to coordinate performance on two separate tasks, the capacity to switch retrieval strategies (for example in random generation tasks) the activation of information held in long-term memory and also selective attention. Another important function of the 
central executive proposed by Morris and Jones [27] is the updating function, namely the ability to modify continuously the content of working memory according to the presentation of newer information. Moreover, it appears quite difficult to find a task in which the role of the CE can be clearly dissociated from that of the slave systems. Some studies have recently used paradigms enabling to isolate precise functions of the CE. D'esposito et al. [11] evaluated cortical areas activated during the concurrent performance of two tasks, which is classically considered to depend on the central executive. An increase in cerebral blood flow was observed in the dorsolateral prefrontal cortex (areas 46 and 9) and in the anterior cingulate gyrus during the dual task only. Salmon et al. [36] investigated the neural basis of another function of the CE, the updating of information maintained in working memory. The updating task requires subjects to watch strings of consonants of unknown length, and then to recall serially a specific number of recent items. Morris and Jones [27] showed that the task requires two independent mechanisms: the updating process requires the central executive but not the phonological loop, and conversely, the serial recall component requires the phonological loop but not the central executive. Salmon et al. [36] contrasted an updating task (requiring both the phonological loop and the central executive components) and a verbal short-term memory task (requiring only the phonological loop) and found an increase of cerebral blood flow in the right mid-dorsolateral prefrontal cortex (BA 9) and frontal pole, as well as in left middle frontal regions (BA 46/10), which were considered to subserve the updating function of the central executive. Other foci of activation were also observed in both inferior parietal regions (BA 40/39), predominantly on the right side. These activations were attributed to the use of visuospatial strategies mixed with the expected verbal strategy, probably promoted by the use of a recognition procedure. In a second study [41], the memory load was reduced in order to remove a possible intervention of the storage function of the $\mathrm{CE}$, and a serial recall was preferred to a recognition procedure in order to minimise the intervention of visual strategies. This time, the updating process was related to an increase of regional cerebral blood flow in a brain area centred on the left frontopolar cortex (BA 10), and extending to left middle frontal areas (BA 46). Given the low memory load, this frontopolar activation was assigned to a specific function of the central executive, i.e., the updating of incoming information.

The objective of the present study was to explore cerebral areas implicated in another function of the central executive, i.e., the manipulation of information, using a paradigm enabling to isolate the manipulation function from the storage requirements of the task. The executive processes of the manipulation tasks which can be considered to be dependent upon the central executive include the inhibition of serial order recall, the extraction of alphabetical order from long-term memory and the checking of that order to rearrange the sequence of items before producing the response [6].

\section{Material and methods}

\subsection{Subjects}

Seven male, European, right-handed volunteers (age range 20-26 years) gave written informed consent to take part in this study, which was approved by the University of Liège Ethics Committee. None had any past medical history nor used any medication.

\subsection{Positron emission tomography scanning}

Scans of regional cerebral blood flow (rCBF) were obtained for each subject using a CTI model 951/31R PET scanner (CTI, Knoxville, TN, USA) with 3D acquisition. The physical characteristics of the tomograph have been described previously [10]. Subjects had an individual thermoplastic face mask for head stabilisation. A transmission scan was acquired for attenuation correction using three rotating sources of $68 \mathrm{Ge}$. Emission scans were reconstructed using a Harming filter at a cut-off frequency of 0.5 cycles per pixel giving a transaxial resolution of $8.7 \mathrm{~mm}$ full width at half maximum and an axial resolution of $5 \mathrm{~mm}$ for each of 31 planes, with a total field of view of $10.8 \mathrm{~cm}$ in this direction.

Volunteers received a $30 \mathrm{~s}$ intravenous infusion of $\mathrm{H}_{2} \mathrm{O}^{15}$ (total activity $6 \mathrm{mCi}$ ) through a left forearm cannula. A dynamic PET scan consisting of two frames was collected over a period of 2 min (background frame duration $30 \mathrm{~s}$, second frame duration $90 \mathrm{~s}$ ). The infusion of $\mathrm{O}^{15}$ labelled water began $10 \mathrm{~s}$ after acquisition start time [22]. Cognitive activation started upon $\mathrm{H}_{2} \mathrm{O}^{15}$ infusion, $20 \mathrm{~s}$ before the second scan. The integrated counts per pixel recorded during the second scan were used as an index of $\mathrm{rCBF}[14,23]$. All subjects underwent 12 consecutive rCBF measurements (three for each experimental and control condition). Eight minutes and forty five seconds elapsed between the scans. 
The cognitive tasks were randomly distributed between subjects, with the exception that a cognitive task was not administered twice in succession.

\subsection{Cognitive tasks}

Subjects performed a modified version of the Alpha span task [6], This task involves the presentation of word lists whose length corresponds to the individual subject's span minus one item. In the first condition, subjects have to recall the presented words in serial order. In the second condition, the words have to be recalled in alphabetical order. The storage requirement being equated between both conditions, the only difference consisted of a central executive intervention during alphabetical recall. In the present experiment, two supplementary conditions were added to compare manipulation with and without storage of information: a serial reading task and an alphabetical reading task, which did not implicate any storage of presented information. A factorial design was thus obtained, with two levels of memory (tasks with and without memory) and two levels of manipulation (tasks with and without manipulation), yielding four task conditions.

Details of the different experimental tasks were the following.

(1) The reading task (no memory and no manipulation): the subject silently read the words presented, without trying to memorise them. At the end of the series, a screen with the words previously presented was displayed and the subjects had to read the word in the presentation order.

(2) The manipulation task (no memory but manipulation): the subject silently read the words presented, without trying to memorise them. At the end of the series, a screen with the words previously presented was displayed and the subject had to read the words in alphabetical order. (3) The word recall task (memory and no manipulation): the subject silently read and memorise words serially presented on a screen. After the last word, a screen with lines of crosses was displayed, and the subject had to recall the words in serial order.

(4) The alpha span task (memory and manipulation): the subject silently read and memorise words serially presented on a screen. After the last word, a screen with lines of crosses was displayed, and the subject had to recall the words in their alphabetical order.

In a pre-test session, a classic word span procedure was administered in order to assess the span level of each subject. Sequences of words were presented to the subject at the rate of one item per second, starting with short sequences of two words. The length of the sequences was progressively increased. Two trials were given by level. If one error occurred on one of these two trials, the subjects were given two additional trials. Subjects were instructed to report items orally in serial order. Testing was interrupted when subjects failed to report correctly two of the four sequences at a particular length. The word span was defined as the longest sequence correctly recalled on $50 \%$ of the trials. Only subjects with a span level of five or six words were selected. They were administered respectively series of four or five words corresponding to their span level minus one. Subjects observed sequences of words ( single capital words in 72 point Helvetica font) presented one at a time on a visual display and they responded aloud. Trials in all condition lasted 14 (four words) or 17.5 (five words) seconds. Each word remained one second on the screen, with a half second interval between words. The response time allocated was eight (four words) or ten (five words) seconds. Subjects' responses were recorded on a tape microphone. Subjects were trained on each cognitive task before the PET session. Three minutes before each acquisition, the instructions were repeated. Each task consisted of eight trials.

It should be noted that even if the storage component is minimal in the alphabetical reading condition (manipulation only), some storage requirements nevertheless exist. Indeed, the subjects do not have to store the word list (as in the recall conditions) but they have to check and memorize continuously which words have or have not been produced in the correct alphabetical order. However, this does not constitute a real problem since such storage requirements similarly exist in the reading and recall manipulation tasks. Consequently, this minimal storage component should not cause any confounding effect in our experimental design.

\subsection{Data analysis}

Image analysis was performed on a SPARC workstation (Sun Microsystems, Surrey, UK using statistical parametric mapping software 96 [17]. Each reconstructed rCBF scan, consisting of 31 primary transverse planes, was interpolated to 52 planes to render the voxels isotropic. The twelve acquisitions from each subject were realigned using the first as reference [43]. The data were then transformed into a standard stereotactic space [39]. A gaussian filter (16 mm full width at half maximum) was applied to smooth each image to accommodate inter subject differences in gyral and functional anatomy and to suppress high frequency noise in the images. Such transformations of the 
data allows for pixel by pixel averaging of data across subjects and for direct cross-reference to the anatomical features in the standard stereotactic atlas.

Differences in global activity within and between subjects were removed by analysis of covariance on a pixel by pixel basis with global count as covariate and regional activity across subjects for each task as treatment [15]. The across-task comparisons were first performed by averaging between paired measurements. For each pixel in stereotactic space, the analysis of covariance (ANCOVA) generated a condition-specific adjusted mean $\mathrm{rCBF}$ value (normalised to $50 \mathrm{ml} / 100 \mathrm{ml} / \mathrm{min}$ ) and an associated adjusted error variance. The ANCOVA allowed the comparison of the means across conditions on a pixel-by-pixel basis using the $t$ statistic. The resulting sets of $t$ values constituted statistical parametric maps $[\mathrm{SPM}(\mathrm{t})][16]$. The SPM(t) were transformed to the unit normal distribution $[\mathrm{SPM}(\mathrm{Z})]$. The design of our study was firstly to evaluate the main effect of manipulation with a comparison of the tasks implicating manipulation of information to tasks implicating serial treatment of information. Secondly, the main effect of verbal working memory was evaluated by comparing reading and recall of the presented information. Finally, the interaction effect between the variables memory and manipulation was also computed. We used a SPM thresholded at $p<0.001$, with further correction for multiple comparisons $(\mathrm{p}<0.05$ ). SPM was thresholded to $p<0.01$ (uncorrected for multiple comparisons), when looking for activation in a region predicted from previous studies.

\section{Table 1 Main effect of manipulation}

Brain area

Stereotactic coordinates Z-score

X $\quad y \quad$ z

$P$-value $<0.001$, with further corrections for multiple comparisons

$\begin{array}{lllll}\text { L inferior/superior parietal (BA 7) } & -28 & -62 & 44 & 6.09 \\ \begin{array}{l}\text { L superior temporal gyrus } \\ \text { (BA22/42) }\end{array} & -58 & -32 & 20 & 4.85 \\ \text { L middle temporal gyrus (BA 21) } & -58 & -12 & 16 & 4.67 \\ \text { R inferior parietal (BA 40) } & 56 & -24 & 20 & 4.84 \\ \text { Posterior cingulate gyrus (BA 31) } & -6 & -40 & 42 & 4.75\end{array}$

$P$-value $<0.01$, non corrected for multiple comparisons

R middle frontal (BA 10/46)

$44 \quad 56$

$38 \quad 50$

$46 \quad 32$

L middle frontal (BA 9/6)

$-36 \quad 18$

$-42 \quad 24$

$-38 \quad 4$

Coordinates and Z-scores for voxels in which there was significant activation or deactivation foci when manipulation was compared to serial treatment of information. Deactivations are shown in italic; $\mathrm{L}=$ left hemisphere; $\mathrm{R}=$ right hemisphere, $\mathrm{x}, \mathrm{y}, \mathrm{z}$ (in $\mathrm{mm}$ ) refer to coordinates in the Talairach space [39].

\section{Results}

\subsection{Neuropsychological performances}

The number of successful trials in each condition (maximum $=24$ ) were the following: reading task: $23.9+0.3$; manipulation task: $21.1 \pm 2.9$; serial recall task: $21.1 \pm 2.6$; alpha span task: $15 \pm 6.41$. The Wilcoxon test showed that subjects had a lower performance in the alpha span task than in the serial recall task $(\mathrm{Z}=1.99, p<0.05)$ and a similar performance in the serial and alphabetical reading $\operatorname{task}(\mathrm{Z}=$ $1.78, \mathrm{NS})$.

\subsection{Imaging data}

\subsubsection{Effect of manipulation}

The predominant activation in the alphabetical tasks occurred in a left parietal area (Brodmann's area or BA 7), just between inferior and superior parietal regions (see Table 1 and Fig. 1). Moreover, we anticipated involvement of prefrontal areas (BA 9/46 and BA 10) when examining the effect of manipulation on brain activation since previous studies have shown that activity in these areas can be related to the central executive functioning [e.g., Refs. [11,36,38]]. Indeed, when the alphabetical tasks were compared to the serial tasks, two brain regions showed a significant main effect of manipulation: the right (BA 10/46) and left middle frontal gyrus (BA 9/6; $p<0.01$, uncorrected for multiple 
comparisons). Deactivation foci were found in the left superior and middle temporal gyrus (BA 22/42 and BA 21), in the right inferior parietal region (BA 40) and in the posterior cingulate gyrus (BA 31).

\subsubsection{Effect of short-term storage}

We next examined the effects of memory on brain activation. We anticipated activation in cortical areas previously related to the phonological loop of working memory, such as the supramarginal gyrus, the Broca area, the temporal cortex, and also the premotor cortex, the anterior cingulate, the parahyppocampal gyrus, the insular area, the thalamus and the cerebellum $[19,31,36]$.

No classical regions devoted to the phonological store or articulatory rehearsal were found (supramarginal gyrus and Broca area), but only a network of 'secondary' regions implicated in shortterm memory showed increases in activity: the cerebellum, the left fronto-insular cortex, the anterior cingulate (BA 32), the right dorsomedial thalamus and the right premotor cortex $(p<0.01$, uncorrected for multiple comparisons; see Table 2).

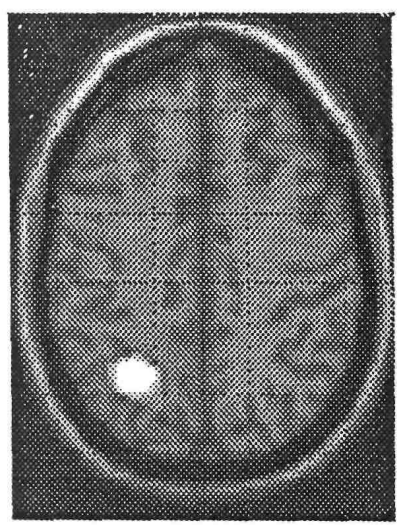

$+44 \mathrm{~mm}$

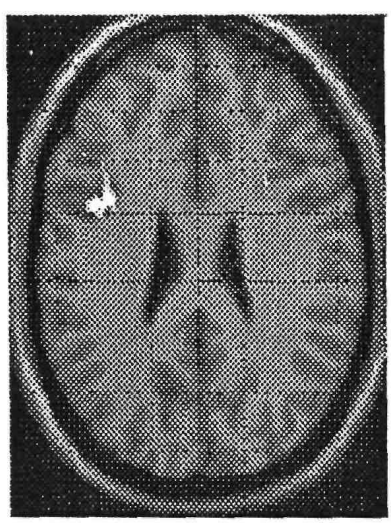

$+26 \mathrm{~mm}$

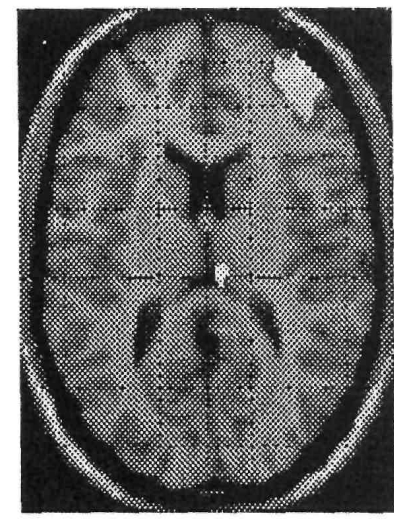

$+12 \mathrm{~mm}$

Fig. 1. Brain activation observed when the manipulation tasks were compared to the serial tasks. Regions with significant $\mathrm{CBF}$ increase are superimposed upon a transverse T1-weighted magnetic resonance imaging slice oriented into the Talairach space [39]. Coordinates of all significant regions are given in Table 1. Note the activity in the left superior parietal $(+44 \mathrm{~mm})$ and the bilateral middle frontal $(+12 \mathrm{~mm}$ and $+26 \mathrm{~mm})$ regions.

Table 2 Main effect of storage

Brain area

$\begin{array}{ll}\text { Stereotactic coordinates } & \text { Z-score } \\ X & y\end{array}$

$P$-value $<0.01$, non corrected for multiple comparisons

Cerebellum

$\begin{array}{llll}-8 & -62 & -30 & 2.81 \\ 4 & -62 & -26 & 2.86 \\ 20 & -56 & -16 & 3.98 \\ -28 & 24 & 12 & 4.04 \\ 6 & 24 & 28 & 3.68 \\ 4 & -14 & 4 & 3.13 \\ 66 & -2 & 24 & 2.98\end{array}$

L fronto-insular

Anterior cingulate (BA 32)

$\mathrm{R}$ dorsomedial thalamus

$\mathrm{R}$ premotor

2.81

4.04

3.68

2.98

Coordinates and Z-scores for voxels in which there was more activation during memory than during reading condition. $\mathrm{L}=$ left hemisphere; $\mathrm{R}=$ right hemisphere, $\mathrm{x}, \mathrm{y}, \mathrm{z}$ (in $\mathrm{mm}$ ) refer to coordinates in the Talairach space [39].

\subsubsection{Interaction between memory and manipulation conditions}

This analysis was performed in order to explore the influence of a memory load on cerebral areas devoted to the manipulation of information. The results showed no significant interaction between these two conditions, indicating that the cerebral areas involved in manipulation of information are similar in the presence or absence of a memory load. In order to confirm the absence of interaction between the factors manipulation and storage, we performed a conjunction analysis of the contrasts alphabetical minus serial in the reading and recall conditions (Table 3). 
Such a conjunction analysis will highlight those regions which are common to the manipulation tasks irrespective of whether or not there is a storage component. Results showed activation foci in the cerebral areas already described in the main effect of manipulation analysis.

Table 3 Conjunction analysis between the constrasts alphabetical-serial in the reading and recall conditions

Area

$\begin{array}{ll}\text { Stereotactic coordinates } & \text { Z-score } \\ X & y\end{array}$

$P$-value $<0.001$, with further corrections for multiple comparisons

$\begin{array}{lcccc}\begin{array}{l}\text { L inferior/superior parietal } \\ \text { (BA7) }\end{array} & -28 & -62 & 44 & 6.48 \\ & -12 & -74 & 50 & 4.34 \\ & & & & \\ P \text {-value }<0.01 \text {, non corrected for multiple comparisons } & & & \\ \text { R inferior parietal (BA 40) } & 42 & -54 & 38 & 4.62 \\ \text { R middle frontal gyrus (BA 10) } & 38 & 50 & 12 & 4.05 \\ & 46 & 32 & 18 & 3.38 \\ & 30 & 56 & 12 & 3.20\end{array}$

Coordinates and Z-scores for voxels in which there was significant activation with the conjunction analysis. $L=$ left hemisphere; $R=$ right hemisphere, $x, y, z$ (in $\mathrm{mm}$ ) refer to coordinates in the Talairach space [39].

Table 4 Manipulation without memory load

Area

$\begin{array}{ll}\text { Stereotactic coordinate: } & \text { s Z-score } \\ \text { X } & y\end{array}$

$P$-value $<0.001$, with further corrections for multiple comparisons

$\begin{array}{lllll}\text { L superior parietal (BA 7) } & -26 & -62 & 44 & 5.43 \\ \text { R superior parietal (BA 7) } & 28 & -74 & 48 & 4.69 \\ \text { Posterior cingulate gyrus (BA 31) } & -6 & -40 & 40 & 5.05 \\ \text { R inferior parietal (BA 40) } & 56 & -24 & 20 & 4.79 \\ \text { L inferior parietal (BA 40) } & -56 & -32 & 20 & 4.70\end{array}$

$P$-value $<0.01$, non corrected for multiple comparisons

$\begin{array}{lllll}\text { R middle frontal gyrus (BA 10) } & 28 & 44 & 6 & 3.17 \\ & 32 & 50 & 12 & 3.49 \\ & 42 & 54 & 16 & 3.95\end{array}$

Coordinates and Z-scores for voxels in which there was significant activation or deactivation when reading in alphabetical order was compared to reading in serial order. Deactivations are shown in italic; $L=$ left hemisphere; $R=$ right hemisphere, $x, y, z$ (in $\mathrm{mm}$ ) refer to coordinates in the Talairach space [39].

\subsubsection{Post-hoc analysis}

The aim of this analysis was to confirm that the manipulation effect is effectively observed in the absence of a memory load. We contrasted regional cerebral activation between serial and alphabetical reading, with the hypothesis that significant activation would be found in similar areas to those described in the main effect of manipulation, namely near the junction of the superior and inferior left parietal area (BA 7), and in the middle prefrontal areas. The results of this post-hoc analysis showed increased $\mathrm{rCBF}$ in the bilateral superior parietal (BA 7) and right middle frontal gyrus (BA 10; $p<0.01$ uncorrected for multiple comparisons) (see Table 4). Deactivation foci were found bilaterally in the inferior parietal (BA 40) and in posterior cingulate gyrus (BA 31).

\section{Discussion}

The aim of the present study was to examine neural networks subserving a function of the central executive, namely the manipulation of information. In order to isolate regions specifically subserving this function, storage requirements were first equated between tasks implicating or not manipulation of information. Moreover, the influence of memory load on CE functioning was also explored by comparing the effect of manipulation on tasks requiring or not storage of information. The regions implicated in manipulation of information were a predominantly left-sided parietal region (BA 7), at the limit between superior and inferior parietal areas, and bilateral middle frontal areas (BA 10/46 and BA 9/6). There was no difference in metabolic activity between tasks requiring manipulation of 
information with and without memory load.

The implication of the prefrontal cortex in tasks assessing the central executive of working memory has been frequently reported in the literature [e.g., Refs. [7,8,11, 32,33,36-38]], with the prefrontal dorsolateral cortex (BA 9/46) being the most frequently activated region, although the implication of other prefrontal areas is commonly observed (e.g., BA 10, Ref. [36]). The activation of different frontal regions during various tasks implicating the central executive can be related to the running of different cognitive processes attributed to that system. However, until now, these different frontal regions were difficult to relate to specific central executive processes because the functioning of the central executive is not sufficiently specified at a theoretical level (see, however, Baddeley [3] for an attempt of fractionation).

We must emphasize that the region most significantly activated by the manipulation of information was a left superior parietal region (BA 7). Increase of regional blood flow in this region has been found in working memory tasks and also in span tasks $[1,33,36-38]$. Since our experimental design was built up to isolate precisely executive functioning from that of the slave systems, the predominant activation of BA 7 should be specifically related to the running of the central executive. The hypothesis of a parietofrontal network sustaining the executive functioning was previously proposed by several authors [12, $18,28,42]$. However, left superior parietal region has also been related to more 'automatic' attentional processes such as directed attention $[9,29,30,34]$. In that context, the frontal and parietal activations observed in the present study would be involved in different aspects of attention, respectively in controlled (frontal) attentional processes (e.g., manipulation of information) and more 'automatic' (parietal) attentional processes (e.g., directed attention to the first letter of the words).

Another interesting finding in this study is the lack of interaction between the variables manipulation and memory. Moreover, a post hoc analysis demonstrated parietal and prefrontal activation in the absence of any storage requirements. These data are indicative that regions implicated in manipulation of information are similar with and without memory load. In other words, the functioning of the central executive is not necessarily related to the presence of a memory load. These results are consistent with the view that the $\mathrm{CE}$ is mainly devoted to executive processes.

The network of brain areas which is activated during the recall tasks does not include the regions classically devoted to the phonological loop, that is the left supra-marginal gyrus related to phonological storage and Broca's area related to articulatory rehearsal [31]. A possible interpretation of the absence of activation in Broca's area is that the inferior frontal cortex is already activated during overt reading tasks. Indeed, the working memory model proposed by Vallar and Cappa [40] links the articulatory rehearsal system to a phonological output buffer involved in speech production which contributes to the articulatory programming of speech output and is involved in working memory tasks as well as in oral production tasks. With regard to the absence of activation in the supramarginal gyrus, previous activation studies suggested that this region is implicated in reading processes [26]. Moreover, the inferior parietal region appears to be associated with the monitoring of oral responses [35].

Finally, deactivation foci are found in tasks implicating manipulation of information in the left superior and middle temporal gyrus, in predominantly the right inferior parietal and in the cingulate posterior gyrus. This decrease of rCBF might be explained by a different allocation of the atten-tional resources according to the cognitive tasks performed. If a given pool of attentional resources is available whatever the task performed, and if a control task does not require all the attentional resources, the remaining resources may be allocated to subsidiary processing. In our experimental tasks, a decrease of $\mathrm{rCBF}$ in the left temporal areas during manipulation may be related to lesser phonological processes [31], and a decrease of $\mathrm{rCBF}$ in the right parietal areas to lesser imagery processes on the presented words $[21,25]$. In a similar way, the decrease of $\mathrm{rCBF}$ in the posterior cingulate gyrus during the experimental conditions may be related to a reduction or removal of transfer of information into longterm memory. Indeed, Fletcher et al. [13] showed the posterior cingulate has a critical role in episodic memory encoding, and that its implication in memory processes was diminished by the simultaneous realisation of a difficult task, probably involving $\mathrm{CE}$ intervention like in our experiment.

\section{Acknowledgements}

This work was supported by the Belgian National Fund for Scientific Research (FNRS), the 'Fondation Medicale Reine Elisabeth', and the Interuniversity Pôle of Attraction Program P4/22, Belgian State, Prime Minister's Office, Federal Office for Scientific, Technical and Cultural Affairs. F. Collette is Aspirant at the FNRS. 


\section{References}

[1] E. Awh, J. Jonides, E.E. Smith, E.H. Schumacher, R.A. Koeppe, S. Katz, Dissociation of storage and rehearsal in verbal working memory: evidence from positron emission tomography, Psychol. Sci. 7 (1996) 25-31.

[2] A. Baddeley, Working Memory, Clarendon Press, Oxford, 1986.

[3] A. Baddeley, Exploring the central executive, Q. J. Exp. Psychol. 49A (1996) 5-28.

[4] JX Becker, MA. Mintum, D.J. Diehl, J. Dobkin, A. Martidis, D.C. Madoff, S.T. DeKosky, Functional neuroanatomy of verbal free recall: a replication study, Hum. Brain Mapp. 1 (1994) 284-292.

[5] J.T. Becker, MA. Mintun, K.D. Aleva, M.B. Wiseman, T. Nichols, S.T. DeKosky, Compensatory reallocation of brain resources supporting verbal episodic memory in Alzheimer's disease, Neurology 46 (1996) 692-700.

[6] S. Belleville, N. Rouleau, N. Caza, Effect of normal aging on the manipulation of information in working memory, Memory and Cognition 26 (1998) 572-583.

[7] J.D. Cohen, W.M. Perlstein, T.S. Braver, L.E. Nystrom, D.C. Noll, J. Jonides, E.E. Smith, Temporal dynamics of brain activation during a working memory task, Nature 386 (1997) 604-607.

[8] J.D. Cohen, S.D. Forman, T.S. Braver, B.J. Casey, D. Servan-Schreiber, D.C. Noll, Activation of the prefrontal cortex in a nonspa-tial working memory task with functional MRI, Hum. Brain Mapp. 1

(1994) 293-304.

[9] M. Corbetta, Frontoparietal cortical networks for directing attention and the eye to visual locations: identical, independent, or overlapping neural system?, Proc. Natl. Acad. Sci. USA 95 (1998) 831-838.

[10] C. Degueldre, L. Quaglia, Performance evaluation of a new whole body position tomograph: the ECAT 951/31 R, Proceedings of the 14th Annual International Conference of the IEEE.EMBS (1992) 1831-1833.

[11] M. D'esposito, J.A. Detre, CD. Alsop, R.K. Shin, S. Atlas, M. Grossman, The neural basis of the central executive of working memory, Nature 378 (1995) 279-281.

[12] M. D'esposito, M. Grossman, The physiological basis of executive function and working memory, The Neuroscientist 2 (1996) 345-352.

[13] P.C. Fletcher, CD. Frith, P.M. Grasby, T. Shallice, R.S.J. Frack-owiack, R.J. Dolan, Brain systems for encoding and retrieval of auditory-verbal memory. An in vivo study in humans, Brain 118 (1995) 401-416.

[14] P.T. Fox, MA. Mintum. Noninvasive functional brain mapping by change-distribution analysis of averaged PET images of $\mathrm{H}_{2}{ }^{15} 0$ tissue activity, J. Nucl. Med. 30 (1989) 141-149.

[15] KJ. Friston, CD. Frith, P.F. Liddle, R.J. Dolan, A.A. Lammertsma, R.S.J. Frackowiak, The relationship between global and local changes in PET scans, J. Cereb. Blood Flow Metab. 10 (1990) 458-466.

[16] K.J. Friston, CD. Frith, P.F. Liddle, R.S.J. Frackowiak, Comparing functional (PET) images: the assessment of significant changes, J. Cereb. Blood Flow Metab. 11 (1991) 690-699.

[17] K.J. Friston, A.P. Holmes, K.J. Worsley, J.-B. Poline, CD. Frith, R.S.J. Frackowiak, Statistical parametric maps in functional imaging: a general linear approach, Hum. Brain Mapp. 2 (1995) 165-189.

[18] J.M. Fuster, Frontal lobes, Curr. Opin. Neurobiol. 3 (1993) 160-165, Review.

[19] P.M. Grasby, CD. Frith, K.J. Friston, C. Bench, R.S.J. Frack-owiack, J.R. Dolan, Functional mapping of brain areas implicated in auditory-verbal memory function, Brain 116 (1993) 1-20.

[20] C. Hulme, S. Roodenrys, R. Mercer, The role of long-term memory mechanisms in memory span, Br. J. Psychol. 86 (1995) 527-536.

[21] S.M. Kosslyn, N.M. Alpert, W.L. Thompson, V. Maljkovic, S.B. Weise, C.F. Chabris et al., Visual mental imagery activates topographically organized cortex: PET investigations, J. Cogn. Neurosci. 5 (1993) 263-287.

[22] A.A. Lammertsma, V.J. Cunningham, M.P. Deiber, J.D. Heather, P.M. Bloomfield, J. Nutt et al., Combination of dynamic and integral methods for generating reproducible functional CBF images, J. Cereb. Blood Flow Metab. 10 (1990) $675-686$.

[23] J.C Mazziotta, S.C. Huang, M.E. Phelps, R.E. Carson, N.S. Mac-

Donald, K. Mahoney, A noninvasive positron computed tomography technique using oxygen-15 labeled water for the evaluation of neurabehavioral task batteries, J. Cereb. Blood Flow Metab. 5 (1985) 70-78.

[24] J.C.D. Mellers, E. Bullmore, M. Brammer, S.C.R. Williams, C. Andrew, N. Sachs, C. Andrews, T.S. Cox, A. Simmons, P. Woodruff, A.S. David, R. Howard, Neural correlates of working memory in a visual letter monitoring task: an fMRI study, Neuroreport 7 (1995) 109-112.

[25] E. Mellet, F. Crivello, N. Tzourio, M. Joliot, L. Petit, L. Laurier et al., Construction of mental images based on verbal description: functional neuroanatomy with PET, Hum. Brain Mapp. Suppl. 1 (1995) 273, Abstract.

[26] M.T. Menard, S.M. Kosslyn, W.L. Thompson, N.M. Alpert, S.L. Rauch, Encoding words and pictures: a positron emission tomography study, Neuropsychologia 34 (1996) 185-194.

[27] R.G. Morris, D.M. Jones, Memory updating in working memory. The role of the central executive, Br. J. Psychol. 81 (1990) 111-121.

[28] R.G. Morris, Working memory in Alzheimer-type dementia, Neuropsychology 8 (1994) 544-554.

[29] A.C. Nobre, G.N. Sebestyen, D.R. Gitelman, M.M. Melusam, R.S J. Frackowiack, CD. Frith, Functional localization of the system for visuospatial attention using positron emission tomography, Brain 120(1997)515-533.

[30] D.S. O'Leary, N.C. Andreasen, R.R. Hurtig, I.J. Torres, LA. Flash-man, M.L. Kesler, S.V. Arndt, T.J. Cizadlo, L.L.B.

Ponto, G.L. Watkins, R.D. Hichwa, Auditory and visual attention assessed with PET, Hum. Brain Mapp. 5 (1997) $422-436$.

[31] E. Paulesu, CD. Frith, R.S.J. Frackowiack, The neural correlates of the verbal component of working memory, Nature 362 (1993) 342-345.

[32] M. Petrides, B. Alivastos, A.C Evans, E. Meyer, Dissociation of human mid-dorsolateral from posterior dorsolateral frontal cortex in memory processing, Proc. Natl. Acad. Sci. USA 90 (1993) 873-877.

[33] M. Petrides, B. Alivastos, E. Meyer, A.C. Evans, Functional activation of the human frontal cortex during the performance of verbal working memory tasks, Proc. Natl. Acad. Sci. USA 90 (1993) 878-882.

[34] M.I. Posner, S. Dehaene, Attentional networks, Trends Neurosci. 17 (1994) 75-79.

[35] C.J. Price, C. Moore, R.SJ. Frackowiak, The effect of varying stimulus rate and duration on brain activity during reading, Neu-roimage 3 (1996) 40-52.

[36] E. Salmon, M. Van der Linden, F. Collette, G. Delfiore, P. Maquet, C. Degueldre, A. Luxen, G. Franck, Regional brain activity during working memory tasks, Brain 119 (1996) 1617-1625.

[37] E.H. Schumacher, E. Lauber, E. Awh, J. Jonides, E.E. Smith, R.A. Koeppe, PET evidence for an amodal verbal working memory system, Neuroimage 3 (1996) 79-88. 
Published in: Cognitive brain research (1999), vol. 7, iss. 3, pp. 411-417

Status: Postprint (Author's version)

[38] E.E. Smith, J. Jonides, R.A. Koeppe, Dissociating verbal and spatial working memory using PET, Cereb. Cortex 6 (1996) $11-20$.

[39] J. Talairach, P. Tournoux, Co-planar stereotaxic atlas of the human brain: 3-dimensional proportional system: an approach to cerebral imaging, Thieme, Stuttgart, 1988.

[40] G. Vallar, S.F. Cappa, Articulation and verbal short-term memory: evidence from anarthria, Cogn. Neuropsychol. 4 (1987) 55-78.

[41] M. Van der Linden, F. Collette, E. Salmon, G. Delfiore, C. Degueldre, A. Luxen, G. Franck, The neural correlates of updating of information in verbal working memory, submitted.

[42] D.R. Weinberger, A connectionist approach to the prefrontal cortex, J. Neuropsychiatr. Clin. Neurosci. 5 (1993) 241-253.

[43] R.P. Woods, S.R. Cherry, J.C Mazziotta, Rapid automated algorithm for aligning and reslicing PET images, J. Comput.

Assist. Tomogr. 16 (1992) 620-633. 